\title{
Coil-Globule Transition of DNA Molecules Induced by Cationic Surfactants: A Dynamic Light Scattering Study
}

\author{
Rita S. Dias, ${ }^{*},+\sharp$ Josef Innerlohinger, ${ }^{\S}$ Otto Glatter, ${ }^{\S}$ Maria G. Miguel, ${ }^{\dagger}$ and Björn Lindman \\ Departamento de Química, Universidade de Coimbra, 3004-535 Coimbra, Portugal, Physical Chemistry 1, \\ Center for Chemistry and Chemical Engineering, Lund University, P.O. Box 124, 22100 Lund, Sweden, and \\ Institut für Chemie, Karl-Franzens Universität Graz, 8010 Graz, Austria
}

Received: December 7, 2004; In Final Form: February 25, 2005

\begin{abstract}
The compaction and aggregation of DNA induced by cationic surfactants was studied by dynamic light scattering (DLS). Furthermore, the effect on surfactant-compacted DNA of the addition of nonionic amphiphiles and salt was studied. When using sufficiently low amounts of DNA and cetyltrimethylammonium bromide (CTAB), compacted DNA molecules could be monitored by the appearance of a band characterized by lower hydrodynamic radius and by the decrease in the intensity of the peak corresponding to extended DNA molecules. Notably, we observed a region where compacted molecules coexist with extended ones; these two populations were found to be stable with time. For higher concentrations of CTAB, only compacted molecules were observed and the size of the particles increased with time indicating aggregation. The number of globules present in the coexistence region increased linearly with the surfactant concentrations, as given by the area of the band corresponding to this population, which indicates a double-cooperativity of the binding. The DLS experiments were in good agreement with previous fluorescence microscopy studies, with certain advantages over this technique since there is no need to add fluorescence dyes and antioxidants. Furthermore, it allows the study of molecules which are too small to be visualized by fluorescence microscopy.
\end{abstract}

\section{Introduction}

The interaction between DNA and cationic surfactants has received, since early times, a great interest from the biomedical sciences. Recently, physical chemists have devoted particular attention to these systems in an attempt to better understand the driving forces behind the molecular interactions; this is also expected to increase the efficiency and number of uses for these systems.

The strong associative behavior displayed by DNA and cationic surfactant systems is well-known and is related to most of its applications such as extraction, purification, and counting. Also gene delivery and transfection constitutes a potential use of these systems. However, synthetic surfactants, like CTAB (cetyltrimethylammonium bromide), cannot be used alone for this purpose because of toxicity and since the complexes between DNA and cationic micelles do not result in effective transfection; but these amphiphiles can be used, in small amounts, for positive charging of neutral liposomes, thus improving their efficiency. ${ }^{1,2}$

Fluorescence microscopy (FM) is one of the most interesting techniques used for the study of these systems, since this technique allows for the direct visualization of single DNA molecules in solution and the study of their conformational behavior in the presence of cosolutes such as surfactants or polyamines. Several studies have been published based on this technique. ${ }^{3-7}$ There are a number of interesting aspects concerning these systems. One is the fact that compaction of DNA is very drastic, that is, the DNA molecule appears either in an extended coil conformation, moving freely in solution with a

\footnotetext{
* Corresponding author. E-mail: rita.dias@fkem1.lu.se.

$\dagger$ Universidade de Coimbra.

$\doteqdot$ Lund University.

$\S$ Karl-Franzens Universität Graz.
}

slow wormlike motion, or in a compacted state, presenting a faster movement and higher fluorescence intensity. Intermediate states are usually not found. Instead, for intermediate concentrations of the cationic surfactants, the two populations coexist in solution. This is a very interesting phenomenon established for DNA molecules on the addition of not only cationic amphiphiles but also flexible polycations, ${ }^{7}$ multivalent ions, ${ }^{6,8,9}$ and organic solvents. ${ }^{10}$

The coil-globule transition of DNA is described as a discrete (quasi-) first-order transition for individual chains but continuous for their ensemble average. ${ }^{9,10}$ Compaction of DNA is driven by attractive interactions between different parts of the molecule due to ion correlation effects arising from a low solvent dielectric constant, ${ }^{10}$ or by the presence of multivalent ions, for example. ${ }^{11-13}$ The compaction is more efficient the higher charged the condensing agent, the maximum degree of compaction being obtained for the interaction with polycations. ${ }^{14}$

Even though surfactant molecules are singly charged they self-assemble in the vicinity of DNA thus acting as multivalent ions. ${ }^{15-17}$ The fact that the compaction of DNA depends on the hydrophobicity of the surfactant is a strong indication for this. ${ }^{4}$

The coexistence of DNA molecules with different conformations in the bulk has only been observed, to our knowledge, by fluorescence microscopy. It has been suggested that conventional techniques, like circular dichroism and light scattering, do not provide a discrete coil-globule transition of the DNA molecules since they monitor the characteristics of the ensemble of chains. ${ }^{18}$ In fact, the conditions at which the coexistence can be observed are very limited in concentration. While for very dilute solutions there are few techniques available which are sensitive enough, when using larger concentrations of DNA and surfactant, for example, a fast aggregation and precipitation of the complexes is obtained, making the study of single molecule compaction impossible. 
Dynamic light scattering (DLS) appeared to be a good technique to study these systems and to present some important advantages over FM experiments. Light scattering techniques are very relevant in the study of colloidal particles, especially in what concerns the size of macromolecules and molecular assemblies. While there have been a number of DLS reports on the properties of DNA solutions (see, for example, refs 1922), studies involving interactions between DNA and cationic amphiphiles are very limited in number. ${ }^{23-25}$ Recently we have reported the possibility of using DLS for studying the coilglobule transition of DNA molecules induced by cationic surfactants. ${ }^{26}$ Here this is further studied with an emphasis on the concentration range for which the coiled and globular conformations coexist.

Considering the most popular applications for DNA-cationic lipid systems we realize that the formation of the complexes is an intermediate step. For nucleic acid purification the desired result is pure DNA; also, in gene delivery DNA must be released from the complexes, after the transfection.

The dissociation of DNA-surfactant complexes in highly diluted solutions was previously studied by the addition of various cosolutes, like monovalent salt, ${ }^{16}$ synthetic polyacid, ${ }^{27}$ neutral liposomes, ${ }^{17}$ and nonionic and anionic surfactants. ${ }^{28,29}$ Anionic species such as multivalent ions and simple surfactants, ${ }^{30}$ as well as negatively charged liposomes, ${ }^{31}$ were shown to dissociate lipoplexes and release DNA into solution.

In this paper we also follow the dissociation of DNAsurfactant complexes by the addition of nonionic surfactants and simple salt.

\section{Experimental Section}

Materials. The surfactants used were cetyltrimethylammonium bromide (CTAB), obtained from Merck and recrystallized three times with an acetone/ethanol mixture, and hexaethylene glycol monododecyl ether $\left(\mathrm{C}_{12} \mathrm{E}_{6}\right)$, from Fluka and used as received. Coliphage T2 DNA $\left(M=1.1 \times 10^{8}\right.$, ca. $\left.164 \mathrm{kbp}\right)$ was purchased from Sigma and phage Lambda DNA $(M=3.15$ $\times 10^{7}$, ca. $48.5 \mathrm{kbp}$ ) from MBI Fermentas. The DNA concentration was determined spectrophotometrically considering the molar extinction coefficient of DNA bases to be equal to 6600 $\mathrm{M}^{-1} \mathrm{~cm}^{-1} .32$ The ratio of the absorbance of the DNA stock solution at $260 \mathrm{~nm}$ to that at $280 \mathrm{~nm}$ was found to be 1.9. $\mathrm{NaBr}$ was obtained from Merck and the Trizma base from Sigma.

Sample Preparation. All stock solutions were prepared in a $10 \mathrm{mM}$ Tris-Cl buffer ( $\mathrm{pH}$ 7.6). DNA, surfactant, and $\mathrm{NaBr}$ stock solutions were prepared by simple dissolution into the desired concentrations. The final concentration of DNA was $0.5 \mu \mathrm{M}$ in nucleotide units. The concentration of cationic surfactant was varied in the compaction studies and the concentrations of nonionic surfactants and salt were varied during the decompaction experiments.

Dynamic Light Scattering: Theory. Dynamic light scattering (DLS), which is also called quasielastic light scattering (QELS) or photon correlation spectroscopy (PCS), is a widely used method for the determination of dynamics and sizes of colloidal systems. ${ }^{33-36}$ It measures the time-dependent fluctuations of the intensity of the scattered light at a fixed scattering angle. The homodyne method, which we use in this work, detects only light scattered from the sample. From this the intensity correlation function $g_{2}(\tau)$ is calculated, which is related to the measured intensities by

$$
g_{2}(\tau)=\lim _{T \rightarrow \infty} \frac{1}{T} \int_{0}^{T} I(t) I(t+\tau) \mathrm{d} t
$$

where $\tau$ is the correlation time. This function is usually normalized to unity for $\tau$ toward infinity. The limit $T$ toward infinity means that the measuring time must be rather long compared to the time scale of the typical movements in the sample. For data evaluation, the field correlation function $g_{1}(\tau)$ is needed. For ergodic samples it can be derived from $g_{2}(\tau)$ by the Siegert relation

$$
g_{1}(\tau)=\sqrt{g_{2}(\tau)-1}
$$

Monodisperse spherical particles show a single-exponential decay in $g_{1}(\tau)$.

$$
g_{1}(\tau)=\mathrm{e}^{-D q^{2} \tau}
$$

The product $D q^{2}$ is the decay rate $\Gamma$. $D$ is the translational diffusion coefficient and $q$ is the length of the scattering vector. It is related to the scattering angle $\theta$ via

$$
q=\frac{4 \pi n}{\lambda} \sin \left(\frac{\theta}{2}\right)
$$

$\lambda$ is the used wavelength in a vacuum and $n$ the refractive index of the sample. From the diffusion coefficient $D$ the hydrodynamic radius $R_{\mathrm{H}}$ can be obtained by using the Stokes-Einstein relation.

$$
D=\frac{k_{\mathrm{B}} T}{6 \pi \eta R_{\mathrm{H}}}
$$

with $\eta$ being the viscosity of the dispersing medium.

For polydisperse samples the simple exponential function in eq 3 is replaced by the weighted contributions of the individual populations. $g_{1}(\tau)$ is, therefore, expressed by the integral

$$
g_{1}(\tau)=\int W(\Gamma) \mathrm{e}^{-\Gamma \tau} \mathrm{d} \Gamma
$$

$W(\Gamma)$ is an intensity distribution function of all decay rates $\Gamma$ contained in the signal. Equation 6 is a Laplace transformation. For the data evaluation, i.e., the determination of $W(\Gamma)$, the distribution function of the diffusion coefficients from a $g_{1}(\tau)$ inversion of this transformation has to be performed. From the distribution of $D$ the distribution of $R_{\mathrm{H}}$ can be easily calculated with eq 5 . The problem is the inverse Laplace transformation, which is a mathematically ill-conditioned problem. There exist software packages which allow the solution of this transformation, like CONTIN ${ }^{37}$ or ORT. ${ }^{38}$

A simpler evaluation is the method of Cumulants. ${ }^{39}$ For a monodisperse sample with one decay, a plot of $\log \left(g_{1}(\tau)\right)$ vs $\tau$ shows a linear behavior. Polydispersity leads to deviations from this simple linear fit. The evaluation is done by series expansion and usually is used to obtain the average size and a polydispersity index.

Experimental Setup and Data Evaluation. For the DLS measurements we use a laboratory built goniometer, which is equipped with a single mode fiber and an ALV single photon detector. The light source is a Verdi V5 diode laser from Coherent with a wavelength of $532 \mathrm{~nm}$ and a maximum output of $5 \mathrm{~W}$. The data acquisition is performed with an ALV 5000 multiple $\tau$ digital correlator, which is combined with an additional fast board. This allows a minimum time interval of $12.5 \mathrm{~ns}$ for the correlation function. The ALV-5000/E software package is used to record and store the correlation functions.

All experiments are carried out at $27^{\circ} \mathrm{C}$ and at a scattering angle of $90^{\circ}$. For one sample $(0.5 \mu \mathrm{M}$ T2DNA plus $2 \mu \mathrm{M}$ 


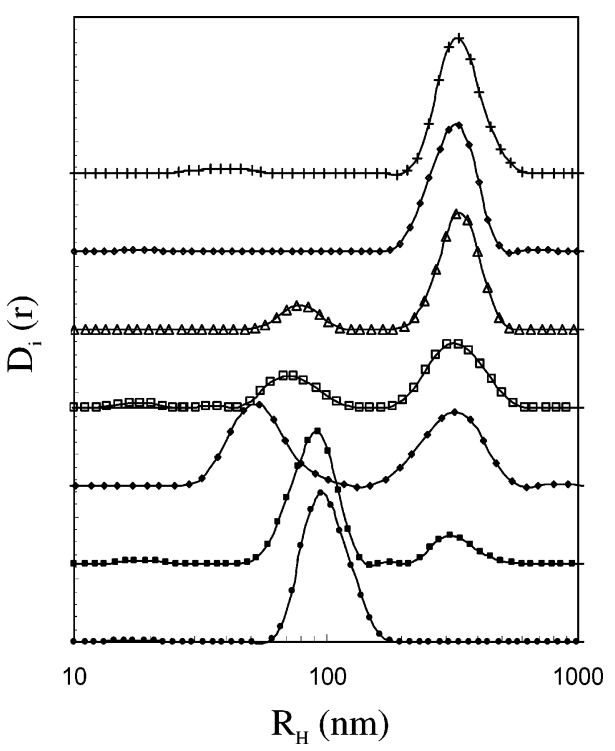

Figure 1. Intensity weighted distribution functions of $0.5 \mu \mathrm{M}$ T2DNA solution in the absence (upper curve) and presence of CTAB. The concentrations of the cationic surfactant are from top to bottom: 0 (only DNA), 1.0, 2.0, 4.0, 6.0, 10.0, and $30.0 \mu \mathrm{M}$. Scattering angle $(\theta)=90^{\circ}$ and $T=27^{\circ} \mathrm{C}$.

$\mathrm{CTAB}$ ) we also performed measurements at $60^{\circ}$ and $105^{\circ}$. This is to control if the second population that we observe does not derive from internal motions of the DNA. The laser power for the experiments is between 0.5 and $1.5 \mathrm{~W}$, depending on the scattering power of the sample. Coiled DNA, being the weakest scatterer, needs the highest power. Each sample is measured 20 times for $40 \mathrm{~s}$. Correlation functions clearly showing some kind of flaw (like from big aggregates passing through the beam) are discarded. The others are averaged and used for calculation of the size distributions. We use the ORT software to obtain the intensity weighted size distributions from the correlation functions.

\section{Results and Discussion}

Compaction of T2DNA by Cationic Surfactants. We started by performing DLS measurements on the DNA and surfactant (CTAB) solutions independently. For the DNA solutions, the average scattered intensity was very low. The DNA used in these experiments has a high molecular weight but its concentration was very low, $0.5 \mu \mathrm{M}$ in phosphate groups, so as to avoid interactions between the molecules. The intensity weighted size distribution of the DNA solution (upper curve in Figure 1) presented only one peak, corresponding to the translational mode of the molecules and resulting in a mean hydrodynamic radius of about $330 \mathrm{~nm}$. For CTAB solutions, the scattering intensity was also very low and we found no significant peaks for solutions with the maximum concentration used in this work.

When the cationic surfactant is added to the DNA solution in a stepwise manner, there are first no detectable changes and then, for a CTAB concentration of $2 \mu \mathrm{M}$, we observe the appearance of a second peak corresponding to entities with a smaller hydrodynamic radius, of about $80 \mathrm{~nm}$. With further addition of CTAB this peak increases in amplitude while the pure DNA translational mode decreases, until it disappears for surfactant concentrations above $30 \mu \mathrm{M}$ (Figure 1).

This is a significant result since it suggests, as observed previously in fluorescence microscopy (FM), not a gradual change of the DNA size but the existence of two populations in the sample, one of extended DNA coils coexisting with DNA compacted molecules.

In Figure $2 \mathrm{a}$ the hydrodynamic radius of the DNA molecules is represented as a function of the surfactant concentration, with data taken from the size distribution calculations (Figure 1). Figure $2 \mathrm{~b}$ shows the long axis of the DNA molecules versus the surfactant concentration as measured directly in FM. The two graphics are quantitatively very similar. As observed for FM the coexistence of two populations of DNA molecules is evident: one due to extended molecules which present some fluctuations in their shape, i.e., size (Gaussian chain), as given by the width of the respective peak; and another consisting of compacted molecules presenting a smaller hydrodynamic radius and less fluctuation in size. The measurements performed by DLS also give an improvement relative to the FM data in what concerns the size of the DNA-surfactant aggregates (note the difference in the scales), since DLS measurements do not need a fluorescent probe, and direct FM measurements overestimate the size of the complexes, due to the blurring effect of the DNA-DAPI complex. ${ }^{27}$ Despite the difference in size one can observe that the ratio between the two populations is the same for both experimental setups. It also can be seen in Figure 2 that the concentration at which DNA compaction starts, as well as the width of the coexistence region, are different with the different techniques. This is probably due to the fact that in the microscopy experiments it is necessary to add an antioxidant, to increase the observation times. This is normally added in relatively large amounts and can thus change the properties of the systems.

Previous DLS measurements on DNA solutions have shown that, ${ }^{20,21}$ for angles of $57^{\circ}$ and higher, the correlation function
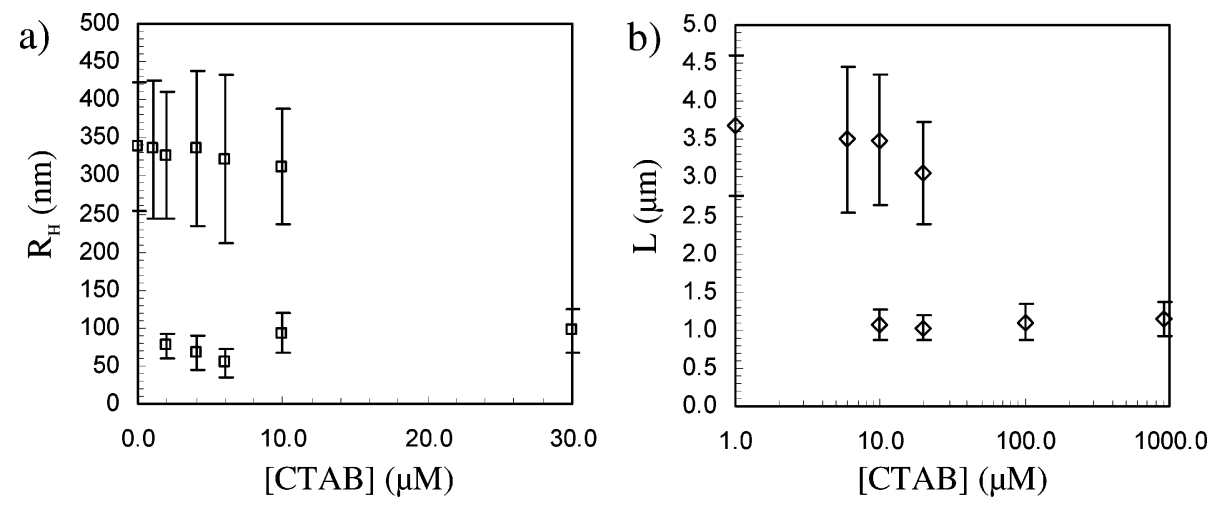

Figure 2. (a) Hydrodynamic radius of the DNA molecules, taken from the position of the peaks of the calculated intensity distribution functions (Figure 1), vs the concentration of surfactant, CTAB. The error bars represent the width of the peak at half-height. (b) Long-axis length, $L$, of DNA molecules, vs the concentration of CTAB, obtained from fluorescence microscopy experiments. Error bars indicate the statistical error in the distribution and are given by the standard deviation. 


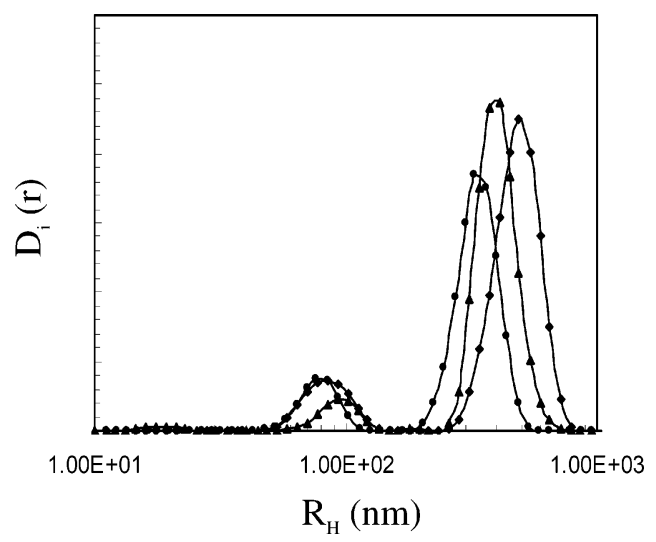

Figure 3. Intensity weighted distribution functions of $0.5 \mu \mathrm{M}$ T2DNA with $2 \mu \mathrm{M} \mathrm{CTAB}$ at different scattering angles: $60^{\circ}$ (diamonds), $90^{\circ}$ (circles) and $105^{\circ}$ (triangles). $T=27^{\circ} \mathrm{C}$.

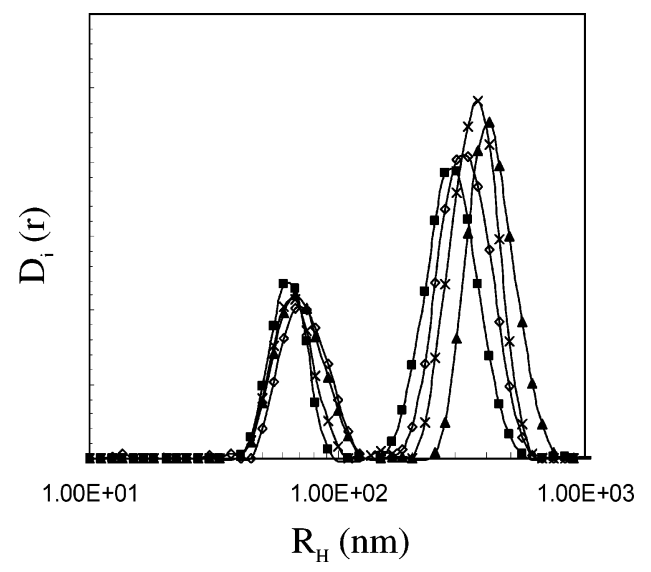

Figure 4. Intensity weighted distribution functions of $0.5 \mu \mathrm{M}$ T2DNA with $4 \mu \mathrm{M} \mathrm{CTAB}$ at different times: 5 (open diamonds), 45 (squares), 75 (triangles), and $830 \mathrm{~min}$ (crosses). Scattering angle $(\theta)=90^{\circ}$ and $T=27^{\circ} \mathrm{C}$.

can exhibit two or more relaxation modes, reflecting internal dynamics in addition to the translational diffusion of DNA. It might be argued that since our measurements were performed at $90^{\circ}$ this could be the case; however, we observed no internal modes. When surfactant is added to the DNA solution, the DNA molecules undergo compaction that leads to a shift in the translational mode of DNA to lower hydrodynamic radius. ${ }^{25}$ If the peak for lower hydrodynamic radius was due to internal modes, the increase in the surfactant concentration would lead to its disappearance, since no internal modes are detected for homogeneous hard spheres; ${ }^{40}$ this is a reasonable assumption for the structure of a compacted DNA molecule. What we observed instead is an increase in the intensity due to the increase in the number of DNA globules in solution. However, to make this point clear, we also performed angular dependence experiments. A concentration of CTAB was chosen in the coexistence region and measurements performed at $60^{\circ}, 90^{\circ}$, and $105^{\circ}$.

As can be seen in Figure 3, the two peaks are present for all three different angles and their positions are essentially invariant. This supports the view that we are in the presence of two different populations.

Stability of the Populations. The stability of the two populations was checked for some samples by measurements at different times. In Figure 4 the size distribution for a sample in the coexistence region is presented, for $[\mathrm{CTAB}]=4.0 \mu \mathrm{M}$, measured after 5, 45, 75, and $830 \mathrm{~min}$. As can be observed the two bands are still visible even after $10 \mathrm{~h}$ and the height ratio

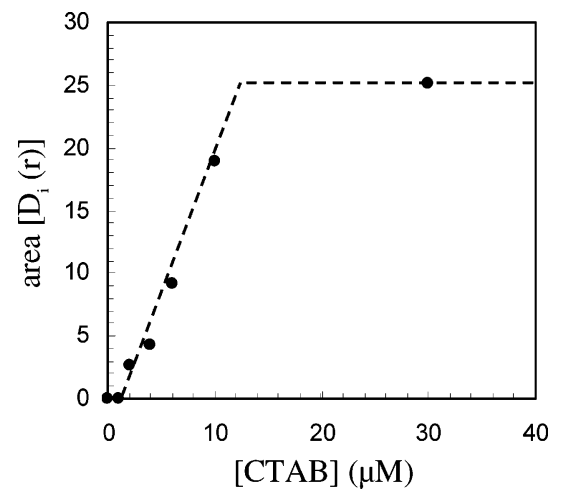

Figure 5. Area of the bands in Figure 1 corresponding to compacted molecules, calculated by numerical integration, vs the concentration of surfactant.

of the two peaks remains essentially constant, indicating that the two populations are stable for at least that period of time.

Experiments were also performed in the globular region. It was observed that in the low concentration part of the globular region, the compacted DNA remains in a single-molecule complex, possibly due to the retaining of some negative charges on the surface of the globules. ${ }^{41}$ However, for higher concentrations of surfactant the globules aggregate and eventually precipitate. Attempts were made to follow the kinetics of aggregation but it was found not to be reproducible enough. For the same concentrations, the aggregation could start immediately or the compacted molecules could remain stable up to 30 or $60 \mathrm{~min}$ before starting to aggregate.

Double Cooperativity. As was mentioned above, the amplitude of the peak corresponding to the extended DNA molecule's translational mode decreases with the addition of surfactant, while the one corresponding to the compacted DNA molecule increases with the addition of cationic surfactants. In Figure 5 we plot the area of the bands corresponding to the compacted molecules in Figure 1 versus the CTAB concentration. We can see that the area under the band increases linearly with the concentration of surfactant until all the molecules are in the compacted state and no more extended DNA is found in solution; then the amount of compacted DNA reaches a plateau. This result demonstrates that the number of globular DNA molecules is directly related to the CTAB concentration. This has already been suggested by the FM studies; however, microscopic techniques are not very reliable since the sampling is somewhat limited. The combination of the two techniques leads clearly to the picture of a double cooperativity in the surfactant binding to DNA.

As mentioned above, the compaction of DNA is dictated by the cooperativity of the surfactant self-assembly in the vicinity of the macromolecule; the surfactant self-assemblies are multivalent counterions which induce electrostatic attractions between different parts of a DNA molecule due to ion correlation effects. ${ }^{11,12}$ In this system there seems to be a different binding situation as compared to systems of more flexible polyelectrolytes. ${ }^{15}$ With DNA we do not observe an even distribution of the surfactant molecules among the DNA molecules and a gradual change in the polymer conformation, but the coexistence of DNA molecules saturated with CTAB and DNA molecules with no significant amount of bound surfactant, as can be seen by the fact that the peak corresponding to extended DNA remains in the same position with the addition of CTAB.

It is the first time, to our knowledge, that the coil-globule coexistence is observed in bulk, in a direct way. Even though FM is an appealing technique for the direct visualization of the 

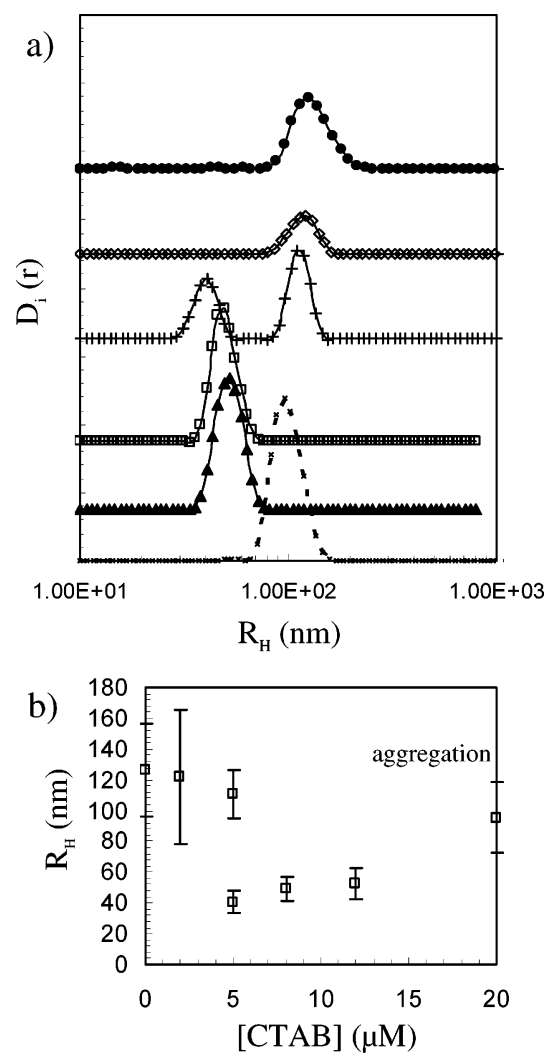

Figure 6. (a) Intensity weighted distribution function of $0.5 \mu \mathrm{M} \lambda \mathrm{DNA}$ solution in the absence (upper curve) and presence of CTAB. The concentrations of the cationic surfactant are from top to bottom: 0 (only DNA), 2.0, 5.0, 8.0, 12.0, and $20.0 \mu \mathrm{M}$. Scattering angle was $90^{\circ}$ and $T=27^{\circ} \mathrm{C}$. (b) Hydrodynamic radius of the DNA molecules, taken from the position of the peaks of the calculated intensity distribution functions in part a, vs the concentration of surfactant, $\mathrm{CTAB}$. The error bars represent, as in Figure 2a, the width of the peak at half-height.

interactions of DNA with cosolutes, we believe that DLS experiments give more reliable information about the sizes of the complexes and the concentrations at which the extended and compacted DNA molecules coexist.

Different DNA Chain Lengths. DLS measurements provide one other advantage when compared with FM studies. They make possible the study of DNA molecules which are long enough to undergo compaction but are too small to be visualized on the optical microscope.

We showed this by performing measurements on $\lambda$ DNA. These molecules with $48.5 \mathrm{kbp}$ have a contour length of only about $16.5 \mu \mathrm{m}$ (against $\sim 56 \mu \mathrm{m}$ of the T2DNA), which makes them too small to be observed by FM and impossible to distinguish between compacted and extended states. Figure 6 shows the evolution of the hydrodynamic radius of the $\lambda \mathrm{DNA}$ molecules with the addition of surfactant. As above a discrete transition of extended DNA molecules to compacted ones is observed, with a coexistence region of the two populations for intermediate CTAB concentrations. The dashed line represents aggregation of the DNA globules, thus observed already for this amphiphile concentration. It is to be noted that, even though the size distributions for these aggregates are very similar to those of extended DNA molecules, it is possible to distinguish them since the scattering of the aggregates is much stronger than the scattering due to DNA coils. The hydrodynamic radius of the macromolecules is represented in Figure $6 \mathrm{~b}$ with the respective fluctuations in sizes. We can see here that the ratio between the extended and the compacted molecules is slightly
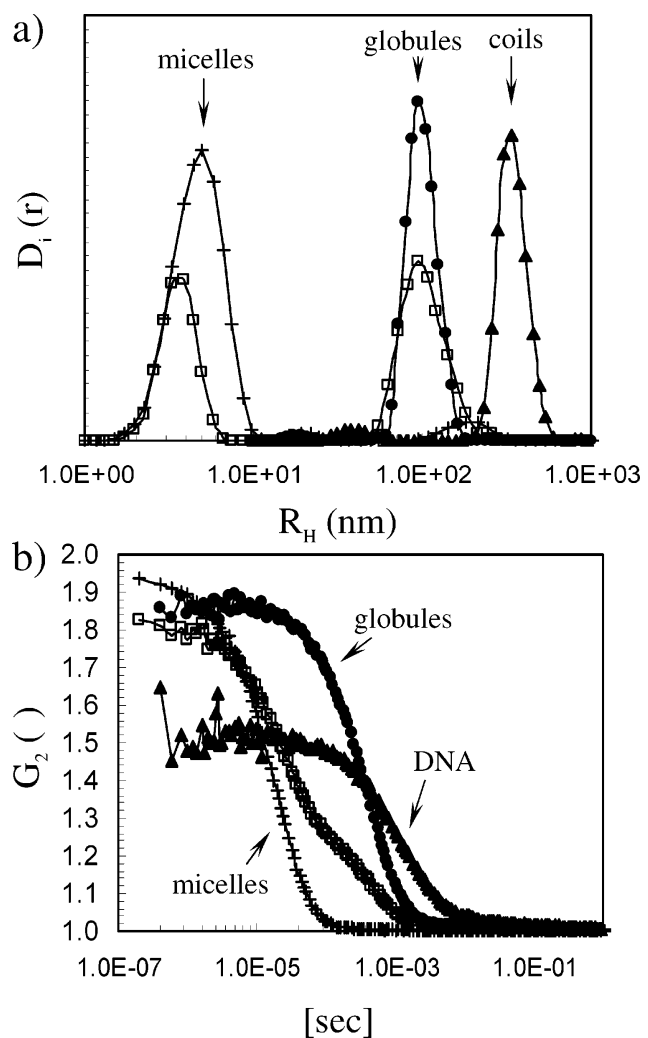

Figure 7. (a) Intensity weighted distribution function for the decompaction studies. T2DNA $(0.5 \mu \mathrm{M})$ is present in all the samples and its distribution function is shown alone for comparison purposes (triangles); the other three samples have $30 \mu \mathrm{M}$ of CTAB and the concentration of nonionic surfactant, $\mathrm{C}_{12} \mathrm{E}_{6}$, is 0 (circles), $1 \mathrm{mM}$ (open squares), and $5 \mathrm{mM}$ (crosses). (b) Correlation functions $G_{2}(\tau)$ of the systems described in part a, using the same symbols. Scattering angle $(\theta)=90^{\circ}$ and $T=27^{\circ} \mathrm{C}$.

smaller in this case than for T2DNA molecules, which can be related to a lower limit in the size of the DNA-CTAB globules.

Decompaction of DNA-Cationic Surfactant Complexes. It is possible to dissociate the DNA-cationic surfactant complexes, and release DNA in solution, by the addition of nonionic surfactants. ${ }^{28} \mathrm{We}$ performed experiments using an oxyethylene surfactant $\left(\mathrm{C}_{12} \mathrm{E}_{6}\right)$ as a decompacting agent. In these experiments a problem arose due to the strong signal of the mixed micelles that were formed in solution; this signal overlapped the comparatively weak signal of DNA alone. The third population, that of the cationic-nonionic mixed micelles, is quite clearly displayed in Figure 7a. In the correlation functions presented in Figure $7 \mathrm{~b}$, for intermediate concentrations of the nonionic surfactant, the two decays corresponding to the mixed micelles and the DNA-cationic complexes in solution are well resolved; however, for higher concentrations of nonionic surfactant where free DNA molecules are considered to appear, only the signal corresponding to the mixed micelles is unambiguously visible.

As was mentioned above, simple salt, when added at relatively high concentrations, can also dissolve the complexes. This approach proved to be most suitable for these experiments since there was no additional complex formation.

In the upper curve of Figure 8 is shown the size distribution for pure DNA. The other curves correspond to samples with DNA and $10 \mu \mathrm{M}$ CTAB, presenting 0 (as control), 5.0, 10.0, and $40.0 \mathrm{mM}$ of $\mathrm{NaBr}$. This concentration of CTAB was used since all the DNA molecules were compacted but was still low enough so that CTAB micelles would not form in solution. It 


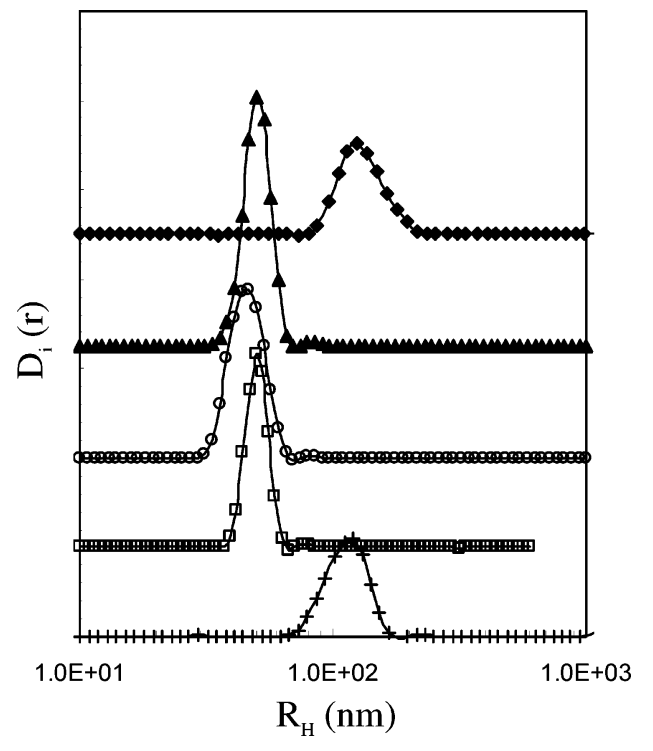

Figure 8. Intensity weighted distribution function for the decompaction studies. The top curve shows data for $\lambda \mathrm{DNA}$ alone (present in all other samples). On the following curves $10 \mu \mathrm{M} \mathrm{CTAB}$ is always present and the concentration of $\mathrm{NaBr}$ is varied, from top to bottom: 0 (only DNA), 0 (DNA $+10 \mu \mathrm{M} \mathrm{CTAB}$ ), 5.0, 10.0, and $40.0 \mathrm{mM}$. Scattering angle was $90^{\circ}$ and $T=27^{\circ} \mathrm{C}$.

is well-known that the CMC of ionic surfactants can decrease up to an order of magnitude with the addition of salt. ${ }^{15}$ It can be seen in the figure that for low concentrations of salt the cationic surfactant remains complexed with DNA, but for relatively high concentrations of $\mathrm{NaBr}(40 \mathrm{mM})$ the DNAcationic surfactant complexes are dissociated and only extended DNA molecules are found in solution. The surfactant is present as monomers since there is no evidence for the formation of CTAB micelles.

\section{Conclusions}

The compaction of DNA molecules, affected by the addition of cationic surfactants, can be directly and conveniently followed by dynamic light scattering. The results are in good agreement with previous fluorescence microscopy experiments. It was observed in a direct way that DNA undergoes a discrete conformational transition from an extended coiled state to a compacted globular one by the addition of cationic surfactants, with a region where the two populations coexist for intermediate concentrations of CTAB. It was shown that in this region the two populations are stable in time, whereas in the globular region, for higher concentrations of surfactant, the DNAsurfactant complexes can aggregate in an apparently nonsystematic manner. The dynamic light scattering presents clear advantages over FM, since there is no need to work with fluorescence dyes and antioxidants, and it also gives the possibility of studying DNA molecules which are too small to be visualized in the optical microscope. It was also possible to follow the dissociation of the DNA-cationic surfactant complexes by the addition of $\mathrm{NaBr}$.

Acknowledgment. This work was supported by grants from Praxis XXI (PRAXIS/BD/21227/99), the Fundação para a Ciência e Tecnologia (FCT) (projects Sapiens POCTI/QUI/ 45344/2002 and POCTI/QUI/45331/2002), the Swedish Research Council (VR), and an EU Research Training Network, CIPSNAC (contract no. MRTN-CT-2003-504932). J.I. and O.G. acknowledge financial support from the Austrian Science
Foundation (FWF) under project P15698 and support from the Marie Curie Network MRTN-CT-2003-504712.

\section{References and Notes}

(1) Pinnaduwage, P.; Schmitt, L.; Huang, L. Biochim. Biophys. Acta 1989, 985, 33-37.

(2) Lasic, D. Liposomes in Gene Delivery; CRC Press: Boca Raton, FL, 1997

(3) Melnikov, S. M.; Sergeyev, V. G.; Yoshikawa, K. Recent Res. Dev. Chem. Sci. 1997, 1, 69-113.

(4) Dias, R.; Mel'nikov, S.; Lindman, B.; Miguel, M. G. Langmuir 2000, 16, 9577-9583.

(5) Makita, N.; Yoshikawa, K. Biophys. Chem. 2002, 99, 43-53.

(6) Yamasaki, Y.; Yoshikawa, K. J. Am. Chem. Soc. 1997, 119, 1057310578

(7) Minagawa, K.; Matsuzawa, Y.; Yoshikawa, K.; Khokhlov, A. R.; Doi, M. Biopolymers 1994, 34, 555-558.

(8) Yoshikawa, K.; Kidoaki, S.; Takahashi, M.; Vasilevskaya, V. V.; Khokhlov, A. R. Ber. Bunsen Phys. Chem. 1996, 100, 876-880.

(9) Yoshikawa, K.; Takahashi, M.; Vasilevskaya, V. V.; Khokhlov, A. R. Phys. Rev. Lett. 1996, 76, 3029-3031.

(10) Mel'nikov, S. M.; Khan, M. O.; Lindman, B.; Jönsson, B. J. Am. Chem. Soc. 1999, 121, 1130-1136.

(11) Guldbrand, L.; Jönsson, B.; Wennerström, H.; Linse, P. J. Chem. Phys. 1984, 80, 2221-2228.

(12) Khan, M. O.; Mel'nikov, S. M.; Jönsson, B. Macromolecules 1999, $32,8836-8840$

(13) Sarraguça, J. M. G.; Skepö, M.; Pais, A. A. C. C.; Linse, P. J. Chem. Phys. 2003, 119, 12621-12628.

(14) Dias, R. S.; Pais, A. A. C. C.; Miguel, M. G.; Lindman, B. J. Chem. Phys. 2003, 119, 8150-8157.

(15) Holmberg, K.; Jönsson, B.; Kronberg, B.; Lindman, B. Surfactants and Polymers in Aqueous Solution, 2nd ed.; John Wiley \& Sons Ltd.: West Sussex, UK, 2003.

(16) Melnikov, S. M.; Sergeyev, V. G.; Yoshikawa, K.; Takahashi, H.; Hatta, I. J. Chem. Phys. 1997, 107, 6917-6924.

(17) Melnikov, S. M.; Sergeyev, V. G.; Melnikova, Y. S.; Yoshikawa, K. J. Chem. Soc., Faraday Trans. 1997, 93, 283-288.

(18) Yoshikawa, K.; Matsuzawa, Y. Physica D 1995, 84, 220-227.

(19) Liu, H.; Gapinski, J.; Skibinska, L.; Patkowski, A.; Pecora, R. J. Chem. Phys. 2000, 113, 6001-6010.

(20) Sorlie, S. S.; Pecora, R. Macromolecules 1990, 23, 487-497.

(21) Sorlie, S. S.; Pecora, R. Macromolecules 1988, 21, 1437-1449.

(22) Borsali, R.; Nguyen, H.; Pecora, R. Macromolecules 1998, 31, $1548-1555$

(23) Guillot, S.; McLoughlin, D.; Jain, N.; Delsanti, M.; Langevin, D. J. Phys.: Condens. Matter 2003, 15, S219-S224.

(24) Gorelov, A. V.; Kudryashov, E. D.; Jacquier, J. C.; McLoughlin, D. M.; Dawson, K. A. Physica A 1998, 249, 216-225.

(25) Cárdenas, M.; Schillén, K.; Nylander, T.; Jansson, J.; Lindman, B. Phys. Chem. Chem. Phys. 2004, 6, 1603-1607.

(26) Dias, R. S.; Pais, A. A. C. C.; Miguel, M. G.; Lindman, B. Colloids Surf., A 2004, 250, 115-131.

(27) Melnikov, S. M.; Sergeyev, V. G.; Yoshikawa, K. J. Am. Chem. Soc. 1995, 117, 9951-9956.

(28) Dias, R. S.; Lindman, B.; Miguel, M. G. Prog. Colloid Polym. Sci. 2001, 118, 163-167.

(29) Dias, R. S.; Lindman, B.; Miguel, M. G. J. Phys. Chem. B 2002, 106, 12608-12612.

(30) Bhattacharya, S.; Mandal, S. S. Biochemistry 1998, 37, 7764-7777.

(31) Xu, Y. H.; Szoka, F. C. Biochemistry 1996, 35, 5616-5623.

(32) Sambrook, J.; Fritsch, E. F.; Maniatis, T. Molecular Cloning: a laboratory manual; Cold Spring Harbor Laboratory Press: New York, 1989.

(33) Berne, B. J.; Pecora, R. Dynamic Light Scattering; John Wiley \& Sons: New York, 1976.

(34) Pecora, R., Ed. Dynamic Light Scattering; Plenum Press: New York, 1985.

(35) Chu, B. Laser Light Scattering, 2nd ed.; Academic Press: San Diego, CA, 1991.

(36) Lindner, P.; Zemb, T. Neutrons, X-rays and Light: Scattering Methods Applied to Soft Condensed Matter; Elsevier Science: Amsterdam, The Netherlands, 2002.

(37) Provencher, S. W. Comput. Phys. Commun. 1982, 27, 213-227.

(38) Schnablegger, H.; Glatter, O. Appl. Opt. 1991, 30, 4889-4896.

(39) Koppel, D. E. J. Chem. Phys. 1972, 57, 4814-4820.

(40) Burchard, W. In Light Scattering. Principles and developments; Brown, W., Ed.; Oxford University Press: Oxford, UK, 1996; pp 439476.

(41) Yamasaki, Y.; Teramoto, Y.; Yoshikawa, K. Biophys. J. 2001, 80, $2823-2832$ 